\title{
Understanding connections between physics and racial Identities through recognition and relational resources
}

\author{
Simone Hyater-Adams, ${ }^{1}$ Claudia Fracchiolla, ${ }^{2}$ Noah Finkelstein, ${ }^{3}$ and Kathleen Hinko ${ }^{2}$ \\ ${ }^{1}$ ATLAS Institute, University of Colorado Boulder, 320 UCB, Boulder, CO, 80309 \\ ${ }^{2} J I L A$, University of Colorado Boulder, 440 UCB, Boulder, CO, 80309 \\ ${ }^{3}$ Physics, University of Colorado Boulder, 390 UCB, Boulder, CO, 80309
}

\begin{abstract}
While there are programmatic efforts to support representation of students from underrepresented groups, as well as studies on student identity, little has been done to link the two. We utilize a framework that combines a racialized identity framework [1] and a physics identity framework [2], in order to understand how identity in physics is impacted by cultural and racial identities. In particular we focus on two dimensions: relational resources [1], which are the relationships that increase one's connection to a practice, and recognition [2], which is recognition as being a good physics student. We operationalize these concepts by analyzing interviews with physicists at the undergraduate student level and beyond. We demonstrate that the constructs of recognition and relational resources overlap through the careful examination of the experiences of two physicists, one who is a black woman and one who is a white man. We discuss early findings that suggest key differences in the role that identity plays in the experiences of physicists of different backgrounds.
\end{abstract}

\section{INTRODUCTION}

The underrepresentation of Black students in physics is well known [3], yet there is still much we do not know about the causes of this problem. Identifying with the culture of a discipline is important when participating in it [4], therefore understanding how students develop an identity in physics is key to cultivating physics students. Historically, physics has been dominated by white men, creating a cultural identity that is partial to participants with this background. To understand what keeps students from different backgrounds in the field, it is necessary to study their experiences in physics culture and how it affects the way they identify with the discipline.

We seek to operationalize and integrate two frameworks, one that defines racialized identity resources [1] and one hat defines physics identity constructs [2], in order to understand how identity in physics is impacted by cultural and racial identities. We foreground the racialized identity framework with the idea that students' racial identities precede any scientific or academic identities, and a physics identity must be negotiated in conjunction to it. We focus on one dimension from each framework: relational resources (relationships that increase connection to a practice) and recognition (as being a physicist). This work builds on our previous study that used the racialized identity [1] framework to study the pathways into physics for volunteers in an after-school program [5]. Here we expand on that study to answer the questions: (1) how do recognition and relational resources overlap and interact with each other to shape a physicist's identity, and (2) how can these constructs give insight to differences in experience for individual physicists?

In this paper, we operationalize the two constructs and test them through the analysis of interviews with a physicist who is a black woman and a physicist who is a white man. We present a two-part work with theoretical contributions operationalizing two frameworks, followed by empirical tests that show how they connect in order to capture qualitative data.

\section{IDENTITY FRAMEWORKS}

Researchers have looked at racial identity and physics identity separately, but only a few have conducted studies about how these identities overlap. We look to build upon these studies by looking at identity within the physics discipline using the work of Nasir [1] who developed a framework of racialized resources that can be available in learning environments, and Hazari et. al. [2] who developed a framework for physics identity.

Racial identity has been studied by many people in different disciplines [6]. Nasir provides a framework to think about racial identity in the context of education. This model examines what she coins as racialized identity through the availability of resources in different learning environments. She defines three broad resource categories: material, ideational, and relational resources. These categories of resources were used to discuss data that showed differences in experience for black high school students.

Additionally, some studies have focused on experiences of students of color in STEM in general that provide tools for looking at science identity [7,8]. Hazari et. al [2] used Carlone and Johnson's [7] study on identity of women of color in STEM to create a discipline-specific framework for physics. This framework includes the constructs of interest, competence, performance, and recognition. The constructs were defined to measure college students' self-perceptions of physics with survey data to examine their correlations.

\section{A. Connecting the Frameworks}

We focus on one aspect of each framework: relational resources (Nasir) and recognition (Hazari et. al). Nasir defines relational resources as the way in which positive relationships with others in the context can increase one's connection to a practice. Hazari et. al's recognition construct is defined as 
recognition from others as being a good physics student. We refine these definitions in order to operationalize them in our analytical process.

Relational resources and recognition are two theoretically linked constructs. While relational resources pertain to how relationships nurture our identity, recognition is usually considered something that comes from relationships. These constructs provide a clear connection between the two frameworks, and an ideal starting point to understand how the frameworks might complement each other. In order to operationalize these two constructs in a coding scheme for interview analysis, we refine the definitions of the constructs and produce subcategories.

\section{B. Methods \& Context}

Drawing from the ideas of narrative inquiry, and the premise that people give meaning to their lives through story [9], we collect narratives of experiences through hour-long interviews. We developed a protocol that prompts interviewees to tell stories about experiences in the physics field, as well as their ideas around what it means to be a physicist. Some key questions from this protocol include: "How did you end up in physics?" and "Can you describe a typical physicist?". Interviewees are asked to expand on their experiences and ideas. The interviews are later transcribed and we use the transcripts to code for the different constructs in the frameworks discussed in the previous section.

Our analysis process includes individual coding, comparison, and group discussion for consensus on code definitions. We started using one interview and the original definitions of the constructs [1][2] to individually code for relational resources and recognition. When collectively discussing this process, it became clear that these codes could be grouped into subcodes. We then coded an additional interview from a very different person to discuss and compare the subcodes. After there was group-wide agreement on the definitions, we collected more interviews to test the analysis process on more data to further refine the codes and inter-rater reliability. We discuss our definitions and the structure of the operationalized frameworks below.

For this paper, we empirically test our operationalized framework on two interviews out of our larger sample of $(n=31)$ in order to demonstrate how the framework picks up the differences in experiences of two different physicists. We intentionally choose two people with very different backgrounds who are at different levels of their career to demonstrate how the framework picks up these differences. The first interview is with Cara, a black woman who has an undergraduate degree in physics and $\mathrm{PhD}$ in Education. The second interview is with Jeff, a senior undergraduate student in physics engaged in atomic, molecular, and optics research, who is a white man.

\section{OPERATIONALIZING RELATIONAL RESOURCES AND RECOGNITION}

Because the recognition and relational resource constructs are specifically defined for the type of analysis done in Nasir and Hazari et. al's work, we refine the original definition for the purpose of this study. Our refined definition of relational resources is "the way in which relationships with others can impact one's connection to a practice". We found that this category can also be defined as positive or negative, depending on whether the relational resources encourage or discourage participation in physics. These codes are operationalized in our interview coding process through the ways that interviewees talk about a significant relationship. A positive relational resource coded in Jeff's interview can be seen when he states "I knew I was going to do something sciencey because both my parents did sciences". An example of a negative relational resource from Cara's interview can be seen when she states "I didn't get along with my science teacher." We define the "unit" of relational resource codes as an aspect of a relationship rather than just the relationships themselves in order to capture the positive and negative impacts that can be attached to a single relationship.

Our refined definition of recognition is "being recognized as a physicist". To capture how recognition is manifested in people's lived experiences, we created subcategories: internal/external and positive/negative. Internal is defined as recognition from one's self, and external is defined as recognition from others. We still define positive and negative as encouraging or discouraging participation in physics. An example of negative internal recognition from Jeff's interview is seen when he states "... I don't think that I'm a personality of a physicist." An example of positive external recognition from Cara's interview is seen when she says "... people who are outside of physics kind of lend me this credit that I wouldn't get unless I was identified with physics." We make these changes to the recognition construct to allow for a more nuanced picture of experiences that differentiates between the impacts of recognition within ones self and from other people. The statements that could not be categorized as positive or negative were collected in an emergent category we name "indeterminate". We discuss in a later section how these codes were able to be linked to the ideational resource construct as defined by Nasir. Table 1 contains the full structure and definitions of our coding scheme, we do not include indeterminate, as it was emergent, and beyond the scope of the initial analysis.

\section{APPLYING THE FRAMEWORK}

The interviews conducted with Cara and Jeff were coded using the operationalized codes and coding process discussed above. We use the interviews from Cara and Jeff, not to make claims about their experiences, but to show how this framework brings out different patterns. We coded interview 
TABLE I. The definitions and structure of the relational resources and recognition codes used in our operationalized framework. The definitions for positive and negative are the same throughout. Codes that could not be categorized as positive or negative were labeled "indeterminate".

\begin{tabular}{|c|c|c|}
\hline $\begin{array}{l}\text { Relational Resource: the } \\
\text { way in which relationships } \\
\text { with others in the context can } \\
\text { increase one's connection to a } \\
\text { practice. }\end{array}$ & \multicolumn{2}{|c|}{$\begin{array}{l}\text { Positive: encouraging participation } \\
\text { in physics. }\end{array}$} \\
\hline \multirow{2}{*}{$\begin{array}{l}\text { Recognition: as being a } \\
\text { physicist. }\end{array}$} & $\begin{array}{l}\text { Internal: from oneself as } \\
\text { being a physicist }\end{array}$ & $\begin{array}{l}\text { Positive } \\
\text { Negative }\end{array}$ \\
\hline & $\begin{array}{l}\text { External: from others as } \\
\text { being a physicist }\end{array}$ & $\begin{array}{l}\text { Positive } \\
\text { Negative }\end{array}$ \\
\hline
\end{tabular}

transcripts first looking for relational resources, and then for recognition. Each relational resource was coded as positive, negative, or indeterminate. All the recognition codes were placed into categories of internal or external, and then labeled as positive, negative, or indeterminate. Table 2 shows the percentages of codes that were in both Cara and Jeff's interviews for each subgroup of codes. Between the two interviews, there were over 200 individual codes, and percentages were normalized to their mother code. For example, the percentage for internal positive recognition is normalized to overall internal recognition.

TABLE II. The percentages of relational resource and recognition subcodes normalized to the overall number of relational resource and recognition codes. The $*$ indicates that there was a statistically significant difference at the $99 \%$ confidence level when a non parametric chi squared test was performed. The positive and negative recognition percentages are normalized to the total number of internal and external recognition codes.

\begin{tabular}{l|l|l|l}
\hline \hline Code & Subcode & Cara & Jeff \\
\hline Relational Resources & Positive & $54 \%$ & $60 \%$ \\
& Negative & $32 \% *$ & $7 \% *$ \\
& Indeterminate & $14 \% *$ & $33 \% *$ \\
\hline Recognition & Internal & & \\
& Positive & $50 \% *$ & $28 \% *$ \\
& Negative & $0 \%$ & $38 \%$ \\
& Indeterminate & $50 \%$ & $38 \%$ \\
\cline { 2 - 3 } & External & & \\
& Positive & $19 \% *$ & $50 \% *$ \\
& Negative & $43 \%$ & $50 \%$ \\
& Indeterminate & $38 \%$ & $0 \%$ \\
\hline \hline
\end{tabular}

The percentages in Table 2 show differences in the numbers of relational resources and recognition for Cara and Jeff's interviews. There were statistically significant differences in percentages for over half of the codes, indicating that the operationalized framework was successful in captur- ing differences in the data. When discussing relational resources, both subjects discussed similar percentages of positive relational resources, but Cara discussed more negative resources in her interview more than Jeff did. There are also differences in the positive/negative subcodes for the different relationships. Cara had negative relational resources from many types of relationships including teachers, peers, advisors, and family. However, Jeff only had negative relational resources from a group of friends and a parent.

Other significant differences show up in the percentages for internal and external recognition. Cara's interview had more discussion of external recognition than internal recognition, where Jeff's interview had the opposite. However, half of the internal recognition codes for Cara were positive, and most of the external recognition codes were negative. Jeff had similar percentages for positive and negative internal recognition, both lower percentages than Cara, as well as equal percentages for positive and negative external recognition. The statistical differences seen in Table 2 served as indicators that led to the identification of the patterns discussed in the next section.

\section{CODING PATTERNS}

After coding the interviews, we identified patterns in the codes that linked relational resources and recognition. These patterns are identified by the ways interviewees express how one construct leads to another in the story of their experiences. The first pattern is shown in Figure 1, where a negative relational resource led to negative external recognition, and caused positive internal recognition. This pattern occurred in both Cara and Jeff's interviews several times. In Jeff's interview, this pattern was an experience where people projected their ideas of a stereotypical physicist on him, and he rejected these ideas by reassuring himself that he was a nonstereotypical physicist. This pattern in Cara's interview includes experiences with people saying and doing things that directly discouraged her from doing physics, causing her to reject their views and continue anyway:"But that professor would not sign off for me to take calculus based physics because he said it would be too hard for me, and he put me in algebra-based physics instead. I wanted to take the class, so I forged his signature on the memo sheet and took the calculusbased physics class and aced it..."

The second pattern shown in Figure 2 emerged from the collection of codes that fit into the "indeterminate" categories under both relational resources and recognition. From the analysis process, we determined that all of the codes that we marked as "indeterminate" were connected to Nasir's ideational resource, which we define as ideas about oneself and one's place in physics, as well as ideas about what is valued and what is good [1]. In both interviews, there were instances where positive relational resources lead to an ideational resource shift that triggered a negative internal recognition. Similar to the last pattern, the stories that held 


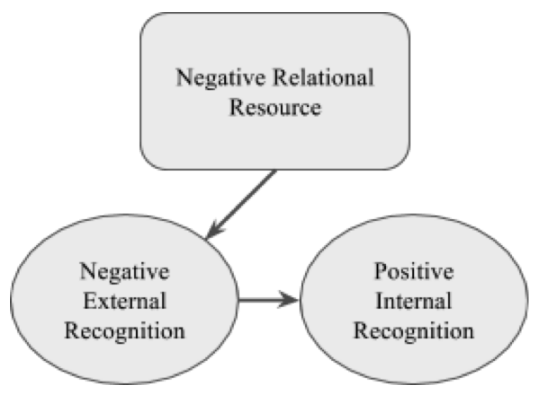

FIG. 1. The first pattern that connects relational resource and recognition codes.

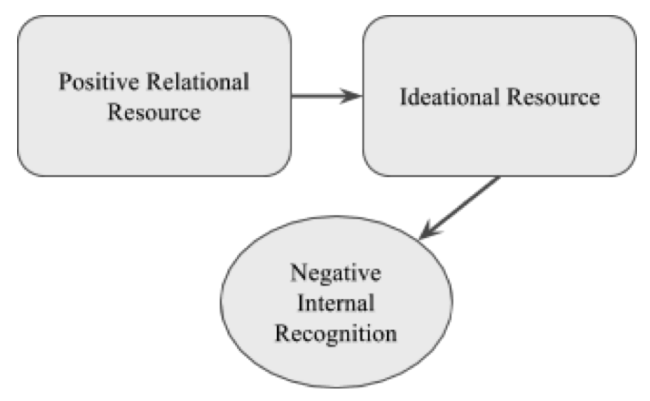

FIG. 2. The second pattern that connects relational resource and recognition codes, that also links to ideational resources.

with this pattern were different for Cara and Jeff. The story Cara told that corresponded with this pattern was about an encouraging mentor she feared to disappoint. Jeff had several instances in his narrative where he acknowledged the discomfort around his privileged position as a white male in physics, and how his relationships with others helped him see that: "It makes me realize that my ability to sort of sail through classes with good grades, and sort of like go down into the lab and not be bothered by anybody, and walk around the halls and be taken seriously, and be able to like joke with people and, you know, just generally do all the things that science people do, I don't have to struggle with that much. And that has completely informed my decision to do science. Because likeAnd I recognize how hard it is for people..." The emergence of the ideational resources in the analysis suggest that our next step in this work should be exploring how they connect to relational resource and recognition.

\section{DISCUSSION \& FUTURE WORK}

From this analysis, we are confident that there may be meaningful connections between the racialized identity and physics identity frameworks because we are able to pull out telling differences between Cara and Jeff's experiences. The operationalized framework captures differences in how frequently they discussed relational resources and recognition, as well as interesting patterns that spanned both interviews. We were also able to see differences in the types of relationships each discussed. Cara talks about several relationships that span from her middle school science teacher to her boss in her current position, where Jeff mentions only his parents, his college advisor, a few friends, and one mentor. However, both Cara and Jeff have broader common patterns that demonstrate the ways that relational resources can interact with recognition empirically.

We identify a limitation of the operationalized framework in capturing how interviewees express the degree of impact that each construct had on their experience. One way this manifested in the data was through the language used in the interview to describe certain experiences. An example of this degree of impact is when Cara emphasizes her experience at a conference for women of color in STEM fields by explaining how she immediately became close friends with women there because of the many shared experiences. The framework fails to bring out this experience as it was emphasized in the narrative. Our future work will include revisiting language analysis used in our previous study [5] to address this limitation in our analysis process.

This work demonstrates potential for addressing a critical gap in the literature: conceptualizing the links between physics identity and racial identity. In its current preliminary stages, it shows promising results that it can pull out differences, as well as commonalities between experiences of two physicists from different racial backgrounds. Our larger goals include operationalizing all of the constructs in the two identity frameworks and conducting the same analysis described in this work to understand how they all connect. Once the framework connections are fully fleshed out, we plan to apply it to interviews from our larger sample to look for more common patterns. From this analysis we will be able to draw conclusions around the differences in physics identity for black students and how these might be emphasized in educational environments. We look to contribute this theoretical work to link theories of identity to intervention strategies.
[1] N. Nasir, Racialized identities. Stan. U.Press, 2011.

[2] Z. Hazari and G. Sonnert,. J. of Res. in Sci. Teach., 2009.

[3] C. Roman and S. Nicholson. "Afri. Amer. Among Deg. Recip. in Physics\&Geosci." AIP Stat. Res. Center, 2010.

[4] E. Wenger "Comm. of prac. and soc. learn. sys." Soc. Learn. Sys and comm of prac. Open U., 2010.

[5] S. Hyater-Adams, K. Hinko, and N. Finkelstein. PERC Proc,
2015 pp. $151-154$

[6] M. McDermott \& F. L. Samson. Whit. Rac. \& Ethn. Id. Ann. Rev. of Soc. Vol 31, 2005.

[7] H. Carlone \& A. Johnson, J.Res.Sci.Teach.,2007 pp.1187- 1218.

[8] M. Ong, Harvard U.. Soc.Prob., 2005, pp. 593-617.

[9] C. McMullen \& I. Braithwaite, J Bus Rsrch Meth, 2013,pp9291. 